\title{
The outbreak of Coronavirus Disease 2019:update on the characteristics and prevention strategies
}

\author{
Kailiang $\mathrm{Xu}^{1}$, Huayong Tan ${ }^{2}$, Yingfeng $\mathrm{Fu}^{2}$, Qian Wang ${ }^{3}$, Hui Liu ${ }^{2}$, Shengbo Han ${ }^{4}$, Ziyang \\ $\mathrm{Di}^{5}$, Dong Zhao ${ }^{6}$, Bo Tang ${ }^{2}$, Yanwei $\mathrm{Liu}^{2}$, Maojun $\mathrm{Di}^{2}$, and QIANG TANG ${ }^{2}$ \\ ${ }^{1}$ Jingzhou Central Hospital \\ ${ }^{2}$ Taihe Hospital \\ ${ }^{3}$ Wuhan Centers for Disease Prevention and Control \\ ${ }^{4}$ Wuhan Union Hospital \\ ${ }^{5}$ Affiliation not available \\ ${ }^{6}$ Wuhan University Renmin Hospital
}

April 28, 2020

\begin{abstract}
In December 2019, with the outbreak of the unidentified pneumonia in Wuhan, China, a novel coronavirus was confirmed to be accountable for this disease and aroused great attention of the entire world. World Health Organization (WHO) officially named the novel coronavirus as SARS-CoV-2 and the unknown pneumonia as Corona Virus Disease (COVID-19). Up to April 13, 2020, there have been 83696 confirmed cases in China and 1837079 cases were totally confirmed in 209 countries outside of China with 119138 fatalities. The main manifestations are usually fever, cough, fatigues, breathlessness, and among others. To date, specific antiviral agents against the SARS-CoV-2 are not available. Extensive measures to reduce person-to-person transmission of COVID-19 have been implemented to control the current outbreak. In this review, we detail current evidence and understanding of the etiology, epidemiology, clinical symptoms and current strategies on diagnosis, treatment, and prevention of COVID-19, which may provide a reference for future studies and help for the prevention and control of the COVID-19 epidemic.
\end{abstract}

\section{Introduction}

In December 2019, an outbreak of unidentified pneumonia characterized by fever, dry cough, and fatigue happened in the Huanan Seafood Wholesale Market, in Wuhan, Hubei, China(J. F.-W. Chan, Yuan, Kok, To, Chu, Yang, Xing, Liu, Yip, \& Poon, 2020; Chen, W, G, \& F, 2020; Hongzhou, W, \& Yi-Wei, 2020). With the spread of the disease, the numbers of infected patients increased substantially which has become the most challenging health emergency in China and even all over the world. Sequence analysis of the coronavirus obtained from 5 patients in Wuhan has shown a structure typical to that of other coronaviruses such as SARS coronavirus and MERS coronavirus(N. Chen et al., 2020; Sohrabi et al., 2020). It also revealed that the new coronavirus has the smallest genetic distance from bat coronavirus, and about $80 \%$ similarity with SARS-CoV, and 50\% similarity with MERS-CoV(P. Sun, Lu, Xu, Sun, \& Pan, 2020). Thereafter, the coronavirus was designated as SARS-CoV-2 and the infected disease was named Coronavirus Disease 2019 (COVID-19) by the World Health Organization (WHO)(Li-Li et al., 2020; Sohrabi et al., 2020; Zhu et al., 2020). In addition, the nosocomial infection was found on January 20, 2020 which suggested that COVID-19 can be transmitted from human to human.

As of April 13, 2020 a total of 83696 cases have been confirmed in China and 1837079 cases reported in 209 countries outside of China. Due to the effective prevention and control measures in China, about 93.5\% patients were cured and discharged and the existing confirmed cases decreased to 2083. However, the 
confirmed cases are still growing rapidly in foreign countries including United State, Italy, Spain, Germany and France, and United State has been particularly affected. With the further recognition of COVID-19 and experience in diagnosis and treatment cumulates, the National Health Commission (NHC) released a consensus about the Diagnosis and Treatment Scheme for Novel Corona Virus Pneumonia which put forward new standard for diagnosis and treatment. The present article is to provide a review of the characteristics of the COVID-19 including the epidemiology, clinical features, pathological changes, diagnosis, treatment, and the experience of prevention and control measures for this disease.

\section{Epidemiology ofCOVID-19}

Since December 2019, the first 27 case of unidentified pneumonia with a history of exposure in the Huanan Seafood Market were reported by the Wuhan Municipal Health Commission(Ashour, Elkhatib, Rahman, \& Elshabrawy, 2020). On Jan 11, 2020, the pathogen of the pneumonia was initially confirmed as a new coronavirus. On January 20, the human-to-human transmission and nosocomial infection were official confirmed firstly(J. F.-W. Chan, Yuan, Kok, To, Chu, Yang, Xing, Liu, Yip, Poon, et al., 2020). And the same day, 4 confirmed cases of 2019-nCoV have been reported from three countries outside of China including Thailand ( 2 cases), Japan (1 case) and the Republic of Korea (1 case). With the epidemic further expanding, daily confirmed case increased to 3892, and then fluctuated to 2022 on February 11. Because of the improvement of diagnosis standard for confirmed cases, there were 14109 new clinically diagnosed cases were reported on February 12, 2020. Since then, the number of daily emerging cases gradually declined. However, the daily emerged cases were increased rapidly in abroad. Data released on February 25, 2020 showed foreign countries had overtaken China in confirmed cases per day for the first time (Figure.1) . Up to April 13, 2020, there were 83696 confirmed cases in China, and 1837079 cases were totally confirmed in 209 countries outside of China (Figure. 2, 3 ). From November 2019 to April 13, 2020, the number of cumulative deaths caused by the COVID-19 was 119138, and the overall case-fatality rate (CFR) was $6.20 \%$ which was lower than that of the SARS (9.60\%) and MERS (34.4\%) (Figure.4, Table.1).

The transmission of infectious diseases must rely on three requirement conditions: sources of infection, routes of transmission, and susceptible hosts(Evans, 2013; Keeling \& Rohani, 2011). A growing body of scientific evidence suggests that COVID-19 is a zoonotic disease as with SARS and MERS, and originated from wild bat(Evans, 2013). And pangolins and snakes were likely to be intermediate hosts of SARS-CoV-2. According to the Diagnosis and Treatment Scheme for Novel Corona Virus Pneumonia (Trial) 7th Edition, close contact with symptomatic cases and asymptomatic cases with silent infection are the main transmission routes of 2019-nCoV infection. It suggested that SARS-CoV-2 can be transmitted through respiratory aspirates, droplets, contacts, and digestive tract transmission remained to be confirmed(Peng et al., 2020). Vertical transmission was sporadically reported in some media but not yet proved(Aldohyan et al., 2019). Reports showed that the basic reproductive values (R0) of COVID-19 were calculated between 2 and 3.5, which means that one patient could transmit the disease to two to three other people. Therefore, SARS-CoV-2 appears to be more infectious than SARS-CoV or MERS-CoV based on R0 values at the early stage of this outbreak(Ying, A, Annelies, \& Joacim, 2020)(Table.1 ). Similar to SARS and MERS, nosocomial transmission was a severe problem or even worse. COVID-19 had posed a difficult challenge to healthcare facilities from both the impact of healthcare-associated transmission and the resource burden of controlling and preventing further spread. It has been reported that a total of 3019 health workers were infected, accounting for $4.17 \%$ of total cases. Unfortunately, $14.8 \%$ of confirmed cases were classified as severe or critical and 5 deaths were observed. In terms of susceptible populations, people are generally susceptible to COVID-19 regardless of age or gender(Surveillances, 2020). $86.6 \%$ of all patients were aged from 30 to79, and the median age of the patients was 47 years. The elderly and those with underlying chronic diseases are more likely to become severe cases(Shen et al., 2020).

\section{The etiology of COVID-19}

Coronavirus is comprised of single-stranded positive RNA virus that belongs to an order Nidovirales, family Coronaviridae, and subfamily Orthocoronavirinae (Jie, Fang, \& Zheng-Li, 2019). Coronavirus can be divided to four genera: $\alpha-, \beta-, \gamma-, \delta$-coronavirus according to the characteristics of serotype and genome $(\mathrm{P}, \mathrm{Xin}, \mathrm{P}$, 
\& Y, 2019). Genome sequences analysis showed that the coronavirus is a new type of coronavirus (SARSCoV-2) and belongs to beta-CoV strain(Li-Li et al., 2020; Ren et al., 2020). A recent study demonstrated that SARS-CoV-2 can survive in human respiratory epithelial cells for 96 hours in vitro(Huang et al., 2020). Current studies have revealed that SARS-CoV-2 shared the same receptors with SARS-CoV and MERS$\mathrm{CoV}$ for invading the host cells(Huang et al., 2020). And the spike (S) protein serves as the main antigenic proteins for binding to angiotensin-converting enzyme 2 (ACE2) receptor and mediates subsequent fusion between the envelope and host cell membranes to aid viral entry into the host cell(X. Xu et al., 2020). In fact, SARS-CoV-2 also shared the same physical and chemical characteristics with SARS-CoV and MERS-CoV. Coronaviruses are sensitive to ultraviolet ray and heat. And it can be killed easily by exposed to 56 for 30 mins, $75 \%$ ethanol, chlorine disinfectant, peracetic acid and chloroform(Duan et al., 2003).

\section{Clinical characteristics ofCOVID-19}

\subsection{Clinical manifestations}

Based on the current epidemiological investigation, the incubation period of the COVID-19 is about 1-14 days(Linton et al., 2020a). The main manifestations are fever, dry cough and fatigue and some patients had nasal congestion, runny nose, myalgia and diarrhea(Linton et al., 2020b). According to the severity of symptoms, COVID-19 can be divided into four classes: mild, moderate, severe, and critical(Zu et al., 2020). Mild patients only presented low-grade fever, mild fatigue or dry cough only. Moderate always characterized by fever, respiratory symptoms, and radiographic features. The severe patients should meet one of these criteria: A. dyspnea B. PaO2/FiO2 $<300 \mathrm{mmHg}$. C. Oxygen saturation $<93 \%$ in ambient air (Z. Sun, Thilakavathy, Kumar, He, \& Liu, 2020). And about half of the patients may develop into critical patients with acute respiratory distress syndrome, septic shock, and coagulation dysfunction within a week(Gorbalenya, 2020). According to the recent survey conducted by China CDC, $80.9 \%$ of the confirmed patients (44672) were mild pneumonia cases, $13.8 \%$ were severe cases, and $4.7 \%$ were critical cases. It had been revealed that the fatality rate of critical patients was $49 \%$ (Wang, Wang, Chen, \& Qin, 2020). Patients with basic diseases such as chronic respiratory disease, cardiovascular disease, diabetes, cancers, would developed into ARDS and worsened in a short period of time even died of multiple organ failure and have a higher case fatality rates than those without. Current reported data revealed that $86.6 \%$ of confirmed patients were aged 30-79 years and 1-2\% of the patients were children and newborns (1.5 months to 17 years), and most of whom had a close contact with infected cases or were family cluster cases. In fact, Infected children might appear asymptomatic or present with fever, dry cough, and fatigue, and few presented with gastrointestinal symptoms including abdominal discomfort, nausea, vomiting, abdominal pain, and diarrhea(Shen et al., 2020). Most of them recovered within 1-2 weeks after disease onset. In general, children always presented with relatively mild symptoms and have a good prognosis(J. F.-W. Chan, Yuan, Kok, To, Chu, Yang, Xing, Liu, Yip, \& Poon, 2020). The clinical course of pregnant women with the COVID-19 is similar to patients of the same age.

\subsection{Laboratory examination}

In the early stage of the COVID-19, white blood cell count is normal or decreased, with decreased lymphocyte count. Some patients may present elevated levels of liver enzymes, lactate dehydrogenase, muscle enzymes and myoglobin. The level of troponin may be increased in critically ill patients. Severe cases showed high level of D-dimer and progressively decreased blood lymphocytes count(Jin et al., 2020). The critical patients often have elevated level of inflammatory factors such as IL2, IL7, IL10, GSCF, IP10, MCP1, MIP1a and TNF- $\alpha$ (Chaolin et al., 2020).

Based upon the evidence from clinical research, the SARS-CoV-2 nucleic acids can be detected in nasopharyngeal swabs, sputum, lower respiratory tract secretions using reverse transcription-polymerase chain reaction (RT-PCR) method(Rothan \& Byrareddy, 2020). Some scholar reported that both IgM and IgG titres were markedly increased in nearly all patients, which was normally considered as a transition from earlier to later period of infection(Zhang et al., 2020). Therefore, they developed IgM and IgG detection methods using a cross-reactive nucleocapsid protein(NP) from another SARSr-CoV Rp3, which is $92 \%$ identical to 2019-nCoV 
NP.

\subsection{Chest imaging}

Radiological examinations are of great importance in the early detection and management of COVID-19. According to current experience, lung imaging manifests can be found earlier than clinical symptoms, so imaging examination is vital in preclinical screening(Sohrabi et al., 2020). Therefore, suspected cases should undertake chest examination as soon as possible.

In early stage, multiple small patchy shadows and interstitial changes were detected in the extra pulmonary zone. And then, it developed into multiple ground-glass infiltration (D. Wang et al., 2020). In severe and critically cases, lung lesions usually involved most commonly 4-5 lobes in the bilateral lower and upper lobes. The first report of COVID-19 patients described that bilateral lung involvement was detected in $80 \%$ of patients, and consolidative pattern changes were always observer in most patients in intensive care unit (ICU), but ground-glass pattern always showed in patients not in the ICU(Bernheim et al., 2020). Shi et al. analyzed the CT changes and found that most patients even the asymptomatic patients showed dynamic changes from focal unilateral to diffuse bilateral ground-glass opacities and then progressed to consolidations within 1-3 weeks(X.-W. Xu et al., 2020). In general, combining assessment of imaging features with clinical and laboratory findings can facilitate diagnosis of COVID-19 pneumonia and evaluate severity of the disease.

\section{Thepathological changes in COVID-19 patients}

A recent study reported the biopsy results from two patients who underwent surgery for malignancy and then were found to have been infected with SARS-CoV-2, which provided first opportunities to study the pathology of COVID-19. It revealed that the lungs of patients exhibited edema, proteinaceous exudate, focal reactive hyperplasia of pneumocytes with patchy inflammatory cellular infiltration, and multinucleated giant cells, but hyaline membranes were not prominent(Tian et al., 2020). This study may describe early phase changes of the lung pathology of COVID-19 pneumonia. After that some academics performed autopsy from 12 dead patients and the results were released by the national health commission. The histopathological changes for different organs are summarized below:

Lung The lungs showed evident multi-pulmonary consolidation, acute interstitial inflammatory infiltrates and congestion in the alveolar septae. The lumina of alveoli and bronchioles were variably filled with proteinrich oedema fluid, erythrocytes, cellular debris and lymphocytes. The exudation cells were mainly mononuclear, macrophages and multinucleated giant cells. Type II alveolar epithelial cells proliferated obviously with Inclusion bodies inside. The blood vessel of the alveolar septum had congestion and edema, in which the infiltration of mononuclears lymphocytics, and intravascular hyaline thrombosis can be seen. Focal hemorrhage and necrosis of the lung tissue caused hemorrhagic infarction. Diffuse interstitial pulmonary fibrosis would be presented with the disease progress. Bronchial epithelial cells were denaturation, necrosis and defluvium. Mucus plugs were visible in the bronchial lumen. Due to over-inflation of the alveoli, a small number of the alveolar septum was broken, or the cysts were formed. SARS-CoV-2 particles could be observed in the cytoplasm of bronchial mucosal epitheliums and type II alveolar epithelial cells under an electron microscope. Immunohistochemical staining showed that some alveolar epitheliums and macrophages were positive for SARS-CoV-2 antigens. RT-PCR was positive for SARS-CoV-2 nucleic acids.

Immune system The volume of spleen decreased significantly and the number of lymphocytes was significantly reduced. There were focal patchy hemorrhages, necrosis and proliferation and phagocytosis of macrophages in the splenic tissue, with atrophy of white pulp lymphoid aggregates. The number of lymphocytes decreased obviously, and necrosis was visible in lymph nodes. Immunohistochemical staining showed that CD4 $+\mathrm{T}$ and CD $8+\mathrm{T}$ cells were reduced in the spleen and lymph nodes. The number of three cell lines in the bone marrow was reduced.

Cardiovascular system There was notable degeneration and necrosis in the myocardial cells, and a few monocytes, lymphocytes and neutrophils infiltrated in the interstitium. Endothelial shedding, endovascular inflammation and thrombosis were visible in the blood vessel. 
Liver and gallbladder The volume of the liver increased and its color was dark red. there are degeneration of hepatocytes, congestion of hepatic sinus, focal necrosis with neutrophil infiltration and microthrombosis, which feature the repeated interchange of these kinds of pathological course. The gallbladder was filled with bile.

Kidney There was proteinaceous exudate in the glomerular cavity and degeneration and necrosis in renal tubular epitheliums. Hyperemia, microthrombus and focal fibrosis can be observed in the renal interstitium.

other organs Within the cerebrum, there was evidence of ongestion, edema, mild neuronophagia and some cases showed neurons degeneration. There were focal necrosis in the adrenal glands. Mucosal epitheliums of the esophagus, stomach and intestine had varying degrees of the degeneration, necrosis and shedding.

\section{Diagnosis ofCOVID-19}

As the number of cases increased rapidly, the first task for the clinical diagnostic workflow is to identify the suspected cases and isolating them immediately, which is critical to cutting off the source of infection. The National Health Commission of China released the Diagnosis and Treatment Scheme for Novel Corona Virus Pneumonia (Trial) Edition. Patients comply any item of the epidemiological history and any two items of the clinical manifestations, or comply 3 items of the clinical manifestations mentioned above can be considered as the suspected cases(Table.2) . Based upon the evidence from clinical research, the detection of SARS-CoV-2 nucleic acids in nasopharyngeal swabs, sputum, lower respiratory tract secretions and SARSCoV-2-specific antibodies (IgM and IgG) may be the final etiology diagnosis for the confirmed case (Table.2 ). In addition, COVID-19 can be divided into four classes: mild, moderate, severe, and critical according to the severity of symptoms (Table 3 ). There are some clinical signs and symptoms closely related to the severity of the confirmed cases (Table 4 ).

\section{Treatment of COVID-19}

The treatment site should be identified according to the disease severity. Confirmed and suspected patients should be isolated in hospitals with applicable protective equipment and special conditions (negative pressure ward). The confirmed cases can be admitted in the same ward, but the suspected cases would be better isolated in a single room to avoid infection. In addition, the critical cases should be treated in ICU as soon as possible.

\subsection{General treatment}

1. Rest in bed, strengthen supportive treatment to ensure sufficient energy.

2. Give effective oxygen therapy in time. Closely monitor blood routine, urine routine, C-reactive protein (CRP), biochemical indicators (liver enzyme, myocardial enzyme, renal function, etc.), coagulation function, arterial blood gas analysis, chest imaging.

3. Antiviral treatment: no effective antiviral drug at present. The following drugs can be used: interferon (IFN)- $\alpha$, ribavirin, chloroquine phosphate, arbidol. Pay Attention to the adverse reactions of the above drugs.

4. Antibiotic treatment: strengthen bacteriological monitoring. Antibiotics should be used in time in secondary bacterial infection reasonably.

\subsection{Treatment of severe and critical cases}

1. The principle of treatment: on the basis of symptomatic treatment, actively prevent and treat complications, treat basic diseases and secondary infection timely.

2. Respiratory support: A. oxygen therapy given by the nasal catheter or mask and assess the effect of oxygen inhalation. B. High flow oxygen therapy or noninvasive mechanical ventilation when the respiratory distress or hypoxemia cannot be alleviated after standard oxygen therapy. Intubation and invasive mechanical ventilation, if the condition does not improve or even worsen. C. Lung protective ventilation strategies: High positive end-expiratory pressure (PEEP) can be appropriately used to keep the airway warm and humid. 
3. Circulation support: based on adequate fluid resuscitation, improve microcirculation, closely monitor the changes of blood pressure, heart rate and urine volume of patients, as well as the surplus of lactate and alkali, and apply hemodynamic monitoring if necessary.

4. Rescue treatment: Rescue treatment: applying lung expansion for patients with severe ARDS. Using extracorporeal membrane oxygenation (ECMO) soon as possible, when the mechanical ventilation has no effect. ECMO indications: A. $\mathrm{FiO}_{2}>90 \%$, oxygenation index is less than $80 \mathrm{mmHg}$ for more than 3-4 hours; B. use VV-ECMO mode for the patients with airway platform pressure [?]35cm $\mathrm{H}_{2} \mathrm{O}$ and simple respiratory failure. if circulatory support is needed, VA-ECM0 mode is selected. When the basic diseases are under control and the cardiopulmonary function shows signs of recovery, the machine withdrawal test can be started.

5. Renal failure and renal replacement therapy: search for the cause of renal function damage. Pay attention to the balance of the fluid, acid-base and electrolyte, and the supplement of heat and trace elements. Continuous renal replacement therapy (CRRT) can be used in the severe patients.

6. Other treatments: Chinese medicine treatment, convalescent plasma therapy, blood purification treatment, immunotherapy and so on.

\section{Management of outpatient department}

With the spread of the epidemic, the number of outpatients was significantly increasing. The outbreak of infectious diseases has become a major concern for Emergency Departments (EDs) worldwide as they are frequently the first point of care irrespective of the severity of symptoms. A series of modifications were applied to the patient pathways while visiting the outpatient department or being admitted as inpatients. Every medical center should encourage patients with mild illness to seek medical treatment through the internet platform to avoid outpatient department crowding and reducing the risk of cross infection. Smartphone application software can be used in remote consultation and online training during the epidemic. Medical staffs may be infected easily by the patients, especially the potential asymptomatic patients that have a wide range of contacts with the medical staff. Therefore, the physician should take precautions before receiving a patient for hospitalization and fully prepared for admission and hospitalization. The hospital wards and medical staff should be dedicated specifically to the confirmed cases to reduce or avoid risk of cross-contamination across patients and health wokers. All of the medical staff wear protective clothing, including goggles, surgical masks, and gloves. Change the gloves and disinfect your hands in time after contact with body fluids or pollutants of patients. At the same time, remind all of the patients and their families to wear masks properly.

Each medical center should strictly implement outpatient appointment system and established a screening procedure to minimize cross infection. During the reception process, all patients should enter the ward in turn and keep a distance of $1 \mathrm{~m}$ and avoid contact with each other. The attending physician should closely monitor the patient's temperature, collect the epidemiological history of the patients, the families, caregivers, and visitors during the previous 14 days and complete the physical examination carefully. It is necessary to be wary of the COVID-19 symptoms such as fever, dry cough and dyspnea. At the same time, we should carefully identify asymptomatic carriers or patients with atypical symptoms (diarrhea, abdominal pain) and distinguish this condition from other viral or bacterial infections diseases. the patients whose family member or caregivers are exposed to any infected person shall be considered as the suspected cases and admitted to a single isolation room for further diagnosis. If the patient is critically ill, ambulance transport shall be launched which was organized by the public health emergency management department and The transport team members should be well trained with the knowledge and skills about prevention and control of COVID-19. It is suggested that blood routine examination (C-reactive protein (CRP) and erythrocyte sedimentation rate (ESR), and normal procalcitonin) and chest imaging examination should be included as a routine examination. And RT-qPCR is known as the gold standard for accurate and fast quantification of nucleic acid sequences. We should actively report suspected or confirmed cases to the experts' committee for further precise treatment. And the suspected or confirmed patients should be isolated immediately according to the regulations on prevention of infectious diseases. 
Equipment or instruments in the consulting room should be strictly disinfected. A growing body of evidence showed that wiping the medical devices by sodium hypochlorite disinfectant can significantly inhibit the activity of the virus. And timely dispose of medical waste to maintain environmental hygiene. The infrastructure of the ventilation system and isolation capacity of rooms was subject to be updated. The ward should keep air circulation and purify in time. After the outpatient service, the consulting room should be sterilized by ultraviolet irradiation or ultra-low volume spray thoroughly.

\section{Prevention and Control}

Since Dec. 2019, the outbreak of novel coronavirus pneumonia began from Wuhan. Hubei Province has brought disaster to the country, and even around the world. WHO declared the COVID-19 outbreak as the sixth public health emergency of international concern, following H1N1 (2009), polio (2014), Ebola in West Africa (2014), Zika (2016), and Ebola in the Democratic Republic of Congo (2019)(Gulland; Sohrabi et al., 2020). And then, the infected disease has been categorized as Class B infectious disease stipulated in the law of the People's Republic of China on the prevention and control of infectious diseases for the first time, and is managed as Class A infectious disease. All of the provincial-level divisions in China mainland have launched the highest level of responding mechanism for major public health emergency which means that the provincial headquarters shall organize and coordinate the emergency response work according to the decision deployment and unified command of the State Council. Therefore, the government, health workers, and the public quickly cooperated globally and entered a state of fighting against the new infectious to prevent its spread. Chinese health departments upgraded their disease prevention and control system by summing up their experience of fighting SARS. We had made notable improvements in surveillance, cases reporting system, multidisciplinary research and cross-sectoral collaboration, and enhance public awareness about the disease.

The focus of the anti-epidemic work should be more inclined to prevention and control. Isolation is still the most effective means of containing COVID-19. Effective surveillance and early identification and diagnosis of COVID-19 is the prerequisite for blocking the source of infections and interrupting the chain of transmission. The health administration departments, medical institutions and social organizations shall provide guidance for patients and close contact families for disease prevention. Comprehensive medical institutions and designated hospital were set up to accept the suspected or confirmed COVID-19 patients and ensure the severe and critical cases can be diagnosed and treated effectively. The government taken compulsory measures to shut down all outbound transportation channels, suspended public transportation restricts and cancels all kinds of congregations consecutively. Avoidance behaviors, such as cancelling or postponing social events, reducing use of public transport, keeping children out of school, keeping away from crowded places and avoiding visiting hospitals due to fear of virus transmission occur frequently during pandemic outbreaks. They also ensured the supply of the clinical supplies, living resources, especially the sufficient supply of surgical masks, disinfectants, and other protective materials on the market. Stations, airports, port and so on gave particular attention to screening procedures to detect the body temperature of passengers entering or leaving the area and implement observation/registration for the suspicious patients. We should strengthen public health surveillance and cases reporting system, and make improvement in multidisciplinary research, cross-sectoral collaboration, hygiene knowledge publicity and laboratory and surveillance capacity.

\section{Psychological intervention of medical staff}

During the spread of the epidemic, COVID-19 prevention and control attracted considerable attention. The highly pathogenic and infectious COVID-19 undoubtedly poses a great challenge to the health of front-line medical staff who were in contact with the source of the disease. According to the existing study results, medical staff were at highly risk of infection during the MERS and SARS outbreaks, with $18.6 \%$ of MERS cases occurring in medical staff and $21 \%$ of SARS cases occurring in medical staff(Chan-Yeung, 2004; Kim et al.). Due to insufficient understanding of the characteristics of pathogenic at the beginning of the epidemic outbreak, a large number of cases occurred among health care workers one after another. At present, more than 3,000 medical workers have been infected in china, and unfortunately, several medical staff have deteriorated and died. In fact, healthcare workers were always infected through the use of endotracheal 
suction and intubation, nasogastric feed,cardiopulmonary resuscitation and high flow-rates of oxygen and so on(Gamage et al., 2005). The high risk presented by these procedures has implications for medical practice and organization of hospital care during the current infectious disease outbreak. Therefore, health workers should not pass up any opportunity to increase their knowledge and practical skills for the sake of personal safety and effective working. We should strengthen our emergency response training according to the Infectious Diseases Prevention Law and Emergency Regulations on Public Health Emergencies. Every staff shall enhance the protection awareness and improve the level of diagnosis and treatment in the course of treatment.

However, with the development of the epidemic, medical personnel would inevitably experience excessive fatigue, decreased immunity, mental stress, and even psychological crisis during the long-term and highintensity rescue process, which seriously endangers their physical and mental health which may reduce work efficiency significantly(S. S. C. Chan et al.). Studies have demonstrated that during the outbreak of SARS in 2003, faced with intense prevention work and increased self-stress, front-line medical staff felt vulnerable, uncertain, life threatening and even suffered from mental illness(Dwosh, Hong, Austgarden, Herman, \& Schabas, 2003). Therefore, we cannot ignore providing psychological counseling and crisis intervention training for the medical staffs. In addition, reasonable schedules, adequate sleep, and appropriate diet and exercise are also the keys to improving the body's immunity and reducing the risk of suffering.

\section{Conclusion}

The outbreak of COVID-19 before the Spring Festival of China, and the massive population flow has challenged the economic, medical and public health infrastructure of China and even all over the world. SARSCoV-2 can be transmitted from person to person and be more infectious than SARS-CoV or MERS-CoV. To date, there is no specific antiviral agents against the virus, and the most effective to prevention and control strategies are early identification, early isolation, early diagnosis, and early treatment. In addition, special attention and efforts to protect or reduce transmission should be applied in susceptible populations including children, elderly people, and health care providers. And appropriate psychological interventions and health education on knowledge for disease prevention and control may be necessary to reduce the risks of infection and heighten the work efficiency greatly. Finally, with the efforts of china and other countries, we will learn more about the pathogenesis of the virus and develop specific vaccines and therapeutic drugs to eliminate the threat of COVID-19.

Acknowledgements: We thank Medjaden Bioscience Limited (HongKong, China) for proofreading this manuscript.

Conflict of interest: None declared.

Ethics Statement: The authors confirm that the ethical policies of the journal, as noted on the journal's author guidelines page, have been adhered to. No ethical approval was required as this is a review article with no original research data.

Funding statement: None.

Data availability statement:The data that support the findings of this study are openly available in National Health Commission of the people's Republic of China at http://www.nhc.gov.cn/xcs/xxgzbd/gzbd_index.shtml, and in World Health Organization in https://who.sprinklr.com/. The data are publicly available with no privacy or ethical restrictions. Data sharing is applicable to this article.

\section{Reference:}

Aldohyan, M., Al-Rawashdeh, N., Sakr, F. M., Rahman, S., Alfarhan, A. I., \& Salam, M. (2019). The perceived effectiveness of MERS-CoV educational programs and knowledge transfer among primary healthcare workers: a cross-sectional survey. BMC infectious diseases, 19 (1), 273.

Ashour, H. M., Elkhatib, W. F., Rahman, M., \& Elshabrawy, H. A. (2020). Insights into the Recent 2019 Novel Coronavirus (SARS-CoV-2) in Light of Past Human Coronavirus Outbreaks. Pathogens, 9 (3), 186. 
Bernheim, A., Mei, X., Huang, M., Yang, Y., Fayad, Z. A., Zhang, N., . . . Li, K. (2020). Chest CT findings in coronavirus disease-19 (COVID-19): Relationship to duration of infection. Radiology, 200463.

Chan-Yeung, M. (2004). Severe Acute Respiratory Syndrome (SARS) and Healthcare Workers. International Journal of Occupational \& Environmental Health, 10 (4), 421-427.

Chan, J. F.-W., Yuan, S., Kok, K.-H., To, K. K.-W., Chu, H., Yang, J., . . . Poon, R. W.-S. (2020). A familial cluster of pneumonia associated with the 2019 novel coronavirus indicating person-to-person transmission: a study of a family cluster. The Lancet, 395 (10223), 514-523.

Chan, J. F.-W., Yuan, S., Kok, K.-H., To, K. K.-W., Chu, H., Yang, J., . . . Yuen, K.-Y. (2020). A familial cluster of pneumonia associated with the 2019 novel coronavirus indicating person-to-person transmission: a study of a family cluster. The Lancet, 395 (10223).

Chan, S. S. C., Leung, G. M., Tiwari, A. F. Y., Salili, F., Leung, S. S. K., Wong, D. C. N., . . . Lam, T. H. The Impact of Work-related Risk on Nurses During the SARS Outbreak in Hong Kong. Fam Community Health, 28 (3), 274-287.

Chaolin, H., Yeming, W., Xingwang, L., Lili, R., Jianping, Z., Yi, H., . . . Bin, C. (2020). Clinical features of patients infected with 2019 novel coronavirus in Wuhan, China. The Lancet (Pre-publis).

Chen, N., Zhou, M., Dong, X., Qu, J., Gong, F., Han, Y., . . . Wei, Y. (2020). Epidemiological and clinical characteristics of 99 cases of 2019 novel coronavirus pneumonia in Wuhan, China: a descriptive study. The Lancet, 395 (10223), 507-513.

Chen, W., W, H. P., G, H. F., \& F, G. G. (2020). A novel coronavirus outbreak of global health concern. The Lancet (Pre-publis).

Duan, S., Zhao, X., Wen, R., Huang, J.-j., Pi, G., Zhang, S., . . . Dong, X.-p. (2003). Stability of SARS coronavirus in human specimens and environment and its sensitivity to heating and UV irradiation. Biomedical and environmental sciences: BES, 16 (3), 246.

Dwosh, H. A., Hong, H. H., Austgarden, D., Herman, S., \& Schabas, R. (2003). Identification and containment of an outbreak of SARS in a community hospital. Cmaj, 168 (11), 1415-1420.

Evans, A. S. (2013). Viral infections of humans: epidemiology and control : Springer Science \& Business Media.

Gamage, B., Moore, D., Copes, R., Yassi, A., Bryce, E., \& Group, B. I. R. P. S. (2005). Protecting health care workers from SARS and other respiratory pathogens: a review of the infection control literature. American Journal of Infection Control, 33 (2), 114-121.

Gorbalenya, A. E. (2020). Severe acute respiratory syndrome-related coronavirus-The species and its viruses, a statement of the Coronavirus Study Group. BioRxiv .

Gulland, A. WHO declares polio a public health emergency. Bmj, 348 (may06 13), g3124-g3124.

Hongzhou, L., W, S. C., \& Yi-Wei, T. (2020). Outbreak of pneumonia of unknown etiology in Wuhan, China: The mystery and the miracle. Journal of medical virology, 92 (4).

Huang, C., Wang, Y., Li, X., Ren, L., Zhao, J., Hu, Y., . . . Gu, X. (2020). Clinical features of patients infected with 2019 novel coronavirus in Wuhan, China. The Lancet, 395 (10223), 497-506.

Jie, C., Fang, L., \& Zheng-Li, S. (2019). Origin and evolution of pathogenic coronaviruses. Nature reviews. Microbiology, 17 (3).

Jin, Y.-H., Cai, L., Cheng, Z.-S., Cheng, H., Deng, T., Fan, Y.-P., . . . Huang, Q. (2020). A rapid advice guideline for the diagnosis and treatment of 2019 novel coronavirus (2019-nCoV) infected pneumonia (standard version). Military Medical Research, 7 (1), 4. 
Keeling, M. J., \& Rohani, P. (2011).Modeling infectious diseases in humans and animals : Princeton University Press.

Kim, C. J., Choi, W. S., Jung, Y., Kiem, S., Seol, H. Y., Woo, H. J., . . . Kim, Y. S. Surveillance of the MERS Coronavirus Infection in Healthcare Workers after Contact with Confirmed MERS Patients: Incidence and Risk Factors of MERS-CoV Seropositivity. Clinical Microbiology \& Infection the Official Publication of the European Society of Clinical Microbiology \& Infectious Diseases, S1198743X16302415.

Li-Li, R., Ye-Ming, W., Zhi-Qiang, W., Zi-Chun, X., Li, G., Teng, X., . . . Jian-Wei, W. (2020). Identification of a novel coronavirus causing severe pneumonia in human: a descriptive study. Chinese medical journal .

Linton, N. M., Kobayashi, T., Yang, Y., Hayashi, K., Akhmetzhanov, A. R., Jung, S.-m., . . . Nishiura, H. (2020a). Epidemiological characteristics of novel coronavirus infection: A statistical analysis of publicly available case data. medRxiv .

Linton, N. M., Kobayashi, T., Yang, Y., Hayashi, K., Akhmetzhanov, A. R., Jung, S.-m., . . Nishiura, H. (2020b). Incubation Period and Other Epidemiological Characteristics of 2019 Novel Coronavirus Infections with Right Truncation: A Statistical Analysis of Publicly Available Case Data. Journal of Clinical Medicine, $9(2), 538$.

P, W. A. C., Xin, L., P, L. S. K., \& Y, W. P. C. (2019). Global Epidemiology of Bat Coronaviruses. Viruses, $11(2)$.

Peng, X., Xu, X., Li, Y., Cheng, L., Zhou, X., \& Ren, B. (2020). Transmission routes of 2019-nCoV and controls in dental practice. International Journal of Oral Science, 12 (1), 1-6.

Ren, Li-Li1, Wang, Ye-Ming2, Wu, Zhi-Qiang4, . . . Cao. (2020). Identification of a novel coronavirus causing severe pneumonia in human a descriptive study. Chinese medical journal .

Rothan, H. A., \& Byrareddy, S. N. (2020). The epidemiology and pathogenesis of coronavirus disease (COVID-19) outbreak. Journal of Autoimmunity, 102433.

Shen, K., Yang, Y., Wang, T., Zhao, D., Jiang, Y., Jin, R., . . . Lin, L. (2020). Diagnosis, treatment, and prevention of 2019 novel coronavirus infection in children: experts' consensus statement. World Journal of Pediatrics , 1-9.

Sohrabi, C., Alsafi, Z., O’Neill, N., Khan, M., Kerwan, A., Al-Jabir, A., . . . Agha, R. (2020). World Health Organization declares Global Emergency: A review of the 2019 Novel Coronavirus (COVID-19). International Journal of Surgery .

Sun, P., Lu, X., Xu, C., Sun, W., \& Pan, B. (2020). Understanding of COVID-19 based on current evidence. Journal of medical virology .

Sun, Z., Thilakavathy, K., Kumar, S. S., He, G., \& Liu, S. V. (2020). Potential Factors Influencing Repeated SARS Outbreaks in China. International Journal of Environmental Research and Public Health, 17 (5), 1633 .

Surveillances, V. (2020). the epidemiological characteristics of an outbreak of 2019 novel coronavirus diseases (COVID-19)-China, 2020. China CDC Weekly, 2 (8), 113-122.

Tian, S., Hu, W., Niu, L., Liu, H., Xu, H., \& Xiao, S.-Y. (2020). Pulmonary pathology of early phase 2019 novel coronavirus (COVID-19) pneumonia in two patients with lung cancer.Journal of Thoracic Oncology .

Wang, D., Hu, B., Hu, C., Zhu, F., Liu, X., Zhang, J., . . . Xiong, Y. (2020). Clinical characteristics of 138 hospitalized patients with 2019 novel coronavirus-infected pneumonia in Wuhan, China. Jama .

Wang, Y., Wang, Y., Chen, Y., \& Qin, Q. (2020). Unique epidemiological and clinical features of the emerging 2019 novel coronavirus pneumonia (COVID-19) implicate special control measures. Journal of 
medical virology .

Xu, X.-W., Wu, X.-X., Jiang, X.-G., Xu, K.-J., Ying, L.-J., Ma, C.-L., . . . Gao, H.-N. (2020). Clinical findings in a group of patients infected with the 2019 novel coronavirus (SARS-Cov-2) outside of Wuhan, China: retrospective case series. $B m j, 368$.

Xu, X., Chen, P., Wang, J., Feng, J., Zhou, H., Li, X., . . . Hao, P. (2020). Evolution of the novel coronavirus from the ongoing Wuhan outbreak and modeling of its spike protein for risk of human transmission. Science China(Life Sciences), 63 (03), 457-460.

Ying, L., A, G. A., Annelies, W.-S., \& Joacim, R. (2020). The reproductive number of COVID-19 is higher compared to SARS coronavirus. Journal of travel medicine .

Zhang, W., Du, R.-H., Li, B., Zheng, X.-S., Yang, X.-L., Hu, B., . . . Shi, Z.-L. (2020). Molecular and serological investigation of 2019-nCoV infected patients: implication of multiple shedding routes. Emerging Microbes 83 Infections, 9 (1), 386-389.

Zhu, N., Zhang, D., Wang, W., Li, X., Yang, B., Song, J., . . . Tan, W. (2020). A Novel Coronavirus from Patients with Pneumonia in China, 2019. New England Journal of Medicine .

Zu, Z. Y., Jiang, M. D., Xu, P. P., Chen, W., Ni, Q. Q., Lu, G. M., \& Zhang, L. J. (2020). Coronavirus Disease 2019 (COVID-19): A Perspective from China. Radiology, 200490.

Figure legend:

Figure 1.The number of Daily emerged confirmed cases of COVID-19 in China and abroad as of April 13, 2020.

Figure 2. The number of cumulative confirmed cases of COVID-19 in China and abroad as of April 13, 2020.

Figure 3. The number of cumulative confirmed cases of COVID-19 in countries outside of China (Top 20) as of April 13, 2020.

Figure 4. The number of cumulative death and the mortality rate of COVID-19 in China and abroad as of April 13, 2020.
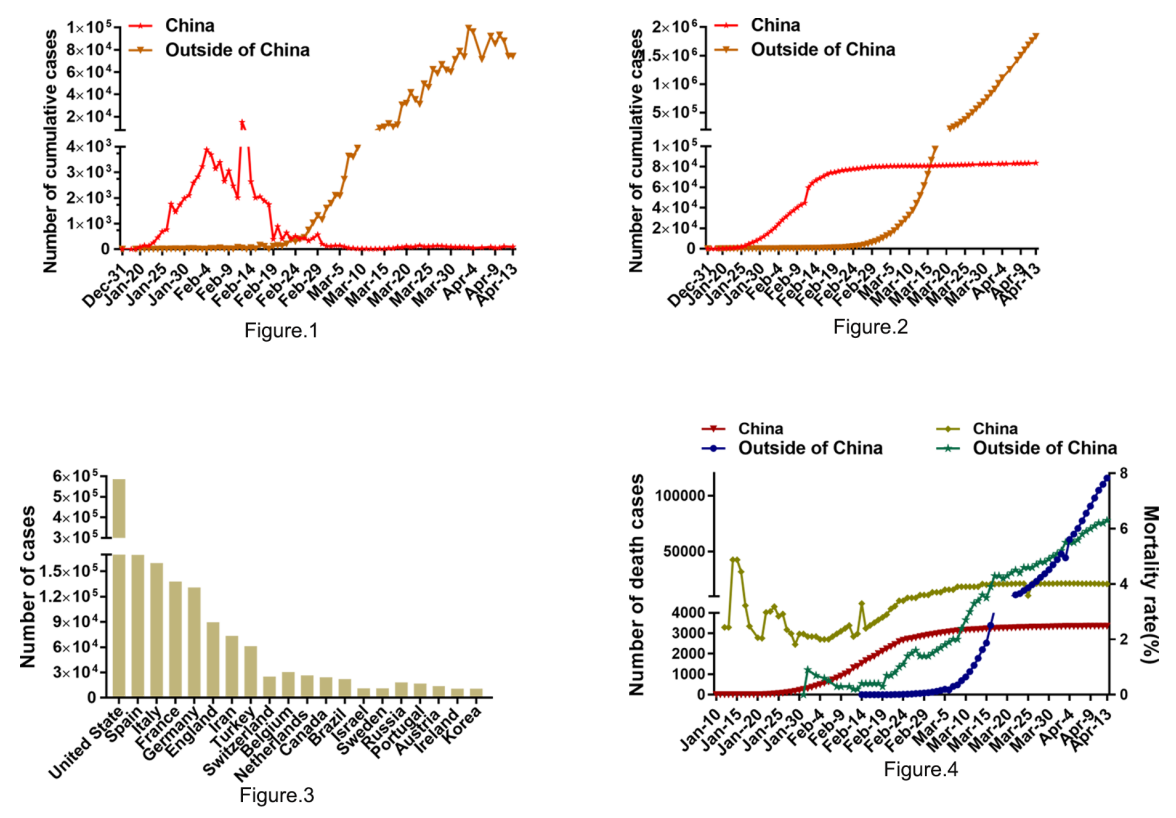


\section{Hosted file}

Tables . docx available at https://authorea. com/users/312854/articles/443413-the-outbreak-of-coronavirusdisease-2019-update-on-the-characteristics-and-prevention-strategies 